\title{
Development and Testing of the Diabetes Self-Management Instrument: A Confirmatory Analysis
}

\author{
Chiu-Chu Lin, ${ }^{1,2} * * *$ Robert M. Anderson, ${ }^{3 \dagger}$ Chao-Sung Chang, ${ }^{4,5}$, \\ Bonnie M. Hagerty, ${ }^{6} *$ Carol J. Loveland-Cherry ${ }^{6 \S}$
}

\author{
${ }^{1}$ Kaohsiung Medical University, College of Nursing, Kaohsiung, Taiwan \\ ${ }^{2}$ Department of Nursing, Kaohsiung Medical University Chung-Ho Memorial Hospital, \\ Kaohsiung, Taiwan \\ ${ }^{3}$ Department of Medical Education, University of Michigan, Ann Arbor, MI \\ ${ }^{4}$ Department of Internal Medicine, Kaohsiung Medical University, Kaohsiung, Taiwan \\ ${ }^{5}$ PingTung Christian Hospital, PingTung, Taiwan \\ ${ }^{6}$ School of Nursing, University of Michigan, Ann Arbor, MI \\ Accepted 19 September 2007
}

\begin{abstract}
The purpose of this study was to develop and psychometrically test a new instrument for measuring self-management of adults with type 2 diabetes. Data were collected from 634 diabetic adults recruited from three teaching hospitals in Taiwan. Construct validity was determined by confirmatory factor analysis (CFA). Tests of internal consistency and testretest were used to assess the reliability of the diabetes self-management instrument (DSMI). Initial results of CFA did not fully support the proposed five-factor model. After the model was modified, the fit indices indicated that this model fits the data best. This model was further cross validated in a second sample. Cronbach's alpha coefficient of the DSMI total scale was .94. The test-retest correlations for the DSMI total scale were acceptable $(r=.73$, $p<.01$ ). ( 2008 Wiley Periodicals, Inc. Res Nurs Health 31:370-380, 2008
\end{abstract}

Keywords: diabetes; self-management instrument; confirmatory analysis

According to the World Health Organization (2004), diabetes will soon reach epidemic proportions in developed countries. Statistics from the United States (U.S.) and Taiwan help to illustrate the problem. In $2005,7 \%$ of the U.S. population had diabetes, and diabetes was among the top
10 causes of death (Centers for Disease Control and Prevention, 2005). Similarly, 4.91\% of the Taiwanese population was diagnosed with diabetes in 2004, and diabetes has become a major cause of death in Taiwan (Taiwan National Department of Health, 2004).

The authors thank Dr. Laura Klem, Dr. Zhong-Ping Cheng, and Miss Chun-Yi Wu for their assistance in statistical analysis.

This study was supported in part by NIH Grant \# 2P60 DK020572 31.

Correspondence to Chiu-Chu Lin, 100 Shih-Chuan, 1st Road, Kaohsiung, Taiwan 807.

*Associate Professor.

**Supervisor.

†'Professor.

${ }^{\ddagger}$ Superintendent.

${ }^{\S}$ Professor, Executive Associate Dean for Academic Affairs.

Published online 22 January 2008 in Wiley InterScience

(www.interscience.wiley.com) DOI: 10.1002/nur.20258 
Because patients provide more than $95 \%$ of diabetes daily care for themselves, successful diabetes management depends largely on their self-management efforts rather than the direct efforts of health care professionals (Funnell \& Anderson, 2000). Consequently, improving diabetes self-management plays a key role in controlling the unwanted complications and sequelae of diabetes.

Diabetes self-management, however, is a complex task that needs to be integrated into the patient's daily life. Successful integration requires the individual to manage this chronic illness in the context of the goals, priorities, health issues, family demands, and personal concerns that make up their lives (Anderson \& Robins, 1998). As they monitor their medications, diet, physical activity, and stress, diabetic patients make day-to-day decisions regarding their illness. Appropriate adjustment of blood glucose levels often involves behavioral change and stringent self-regulation efforts (Gonder-Frederick, Cox, \& Ritterband, 2002; Hill-Briggs, 2003; Petrie, Broadbent, \& Meechan, 2003).

In addition, psychosocial barriers make managing diabetes a struggle for many patients. For example, in Taiwan people with diabetes often face social stigmatization (Lin, Anderson, Hagerty, \& Lee, in press). Self-management of diabetes thus involves an array of physiological and psychosocial issues. In addition, diabetic patients must obtain the support of health care providers, families, friends, and employers (Lin et al.) in the management of their disease.

Because self-management is the cornerstone of diabetes care, it is important to have instruments that accurately evaluate self-management practices. Three problems complicate evaluating self-management practices. First, the literature does not reflect a uniform terminology (Schilling, Grey, \& Knafl, 2002). A literature search revealed that many investigators use the terms self-management, selfcare, and compliance/adherence interchangeably. Furthermore, many authors do not clearly define what they mean by self-management.

Second, most studies of diabetes self-management emphasize glycemic control, while ignoring other aspects of disease management. The self-management of diabetes goes well beyond managing blood glucose levels (i.e., blood glucose monitoring and taking medications); it usually requires adjustments in other areas of the patient's life, such as work, recreation, meals, relationships, and self-image (Lin et al., in press; Price, 1993). Accordingly, selfmanagement should be approached as a multidimensional concept that combines biological, psychological, and social activities (Barlow, Wright, Sheas, Turner, \& Hainsworth, 2002). Lin (2005) identified the dimensions of self-management as illness adaptation, decision making, and illness control through concept synthesis.

Third, in many studies on diabetes the authors fail to use psychometrically tested instruments to measure components of self-management. To test applications of diabetes self-management adequately, investigators must develop or refine instruments to measure the underlying theoretical concepts and to test theoretical relationships (Whittemore, 2000).

Instruments designed to measure self-management should reflect the underlying model upon which they are based. Whittemore (2000) argued that the development of models that clearly illustrate the relationships among variables specific to diabetes self-management is crucial to the advancement of diabetes knowledge. These models must explicitly define diabetes concepts that are related within a meaningful theoretical perspective (Fain, Nettles, Funnell, \& Prochownik, 1999).

In the study reported here, we conceptualized diabetes self-management as an active, flexible process in which patients develop strategies for achieving desired goals by regulating their own actions, collaborating with health care providers and significant others, and performing preventive and therapeutic health-related activities. This view of self-management was derived from Lin's (2005) three dimensions of self-management. The purpose of this study was to develop and test the psychometric properties of a new instrument for measuring self-management behaviors among Taiwanese adults with type 2 diabetes. Two specific aims guided this investigation:

1. To generate items for a diabetes self-management instrument (DSMI) in Chinese.

2. To evaluate the developed DSMI for content, face, and construct validity; internal consistency; and test-retest reliability.

\section{METHODS}

\section{Instrument Development}

Item generation. We constructed an initial draft of the DSMI in English by identifying and sampling the content domain for the indicators 
of diabetes self-management. Sources used to generate the initial item pool included: a comprehensive literature review related to diabetes selfmanagement, focus group findings (Lin et al., in press), and clinical experience. Additionally, a diabetes educator in Taiwan gathered examples of diabetes self-management from seven of her diabetic patients using Lin's (2005) semi-structured questions for each of her three dimensions of diabetes self-management. The examples provided by the Taiwanese patients were used to generate additional items for the instrument. Through this process, 63 candidate items were generated in English to form an initial draft of the DSMI. The draft was then evaluated for content and face validity.

Determination of content validity. Once the pool of candidate items was developed, we assembled a panel of seven experts in diabetes and instrument development. These experts included three doctorally prepared diabetes educators with expertise in instrument development, two physicians specializing in diabetes, and two nurse practitioners who work in a diabetes clinic. The experts were asked to evaluate the initial items' relevance to their associated concept domains based on the conceptual definition provided. The panel assessed each item using a 4-point Likert-type scale: 1-not relevant, 2somewhat relevant, 3-quite relevant (relevant but needs minor alteration), 4-very relevant (Lynn, 1986). If an expert panel member rated any item below 4, the expert was asked to provide his or her suggestions for modifying or eliminating the item.

We used the content validity index (CVI) to quantify the extent of expert agreement. The CVI for an item is the proportion of experts who rate it as a 3 or 4 (Waltz, Strickland, \& Lenz, 2005). The expert panel's assessment resulted in a total CVI score of .90. Eleven additional items were added to the initial draft of the DSMI based on suggestions from four of the seven experts. The other three experts provided only wording suggestions. Problematic items were revised or clarified based on the recommendations from the panel. This resulted in a final draft of 74 items.

Translation from English into Chinese. After assessing the content validity of the English version, investigators used a back-translation method (Carlson, 2000) to obtain a linguistically equivalent instrument in Chinese. This involved first translating the draft into Chinese. A bilingual translator who was a master's-prepared clinical nurse practicing in the U.S. did this translation. Another bilingual translator then translated the
Chinese tool back into English. This second translator was an internal medicine physician in Taiwan. Investigators then compared the original version and the back-translated version to identify discrepancies. Where there were differences between the original and back-translated English versions, investigators worked with the translators to identify the reasons for the discrepancies and to clear up inconsistencies. The Chinese draft was modified accordingly. This resulted in an initial draft of the DSMI-74 in Chinese for use in the Taiwanese diabetic population.

Determination of face validity. To evaluate the face validity of the DSMI, investigators administered this initial Chinese draft of the instrument (i.e., DSMI-74) to a convenience sample from the three data collection sites used in this study. Fifteen Taiwanese volunteers with type 2 diabetes agreed to complete the instrument, simultaneously reviewing it for clarity, item comprehension, and response ease. The volunteers also rated each item to indicate the frequency with which they performed the self-management practices. Items were scored on a 4-point scale ranging from 1 (never) to 4 (always). Feedback from this pre-testing guided the investigators as they made several minor adjustments to the DSMI to better reflect cultural nuances. The pre-testing yielded a 74-item instrument in Chinese that was ready for pilot testing.

\section{Pilot Testing}

We conducted a pilot study using a convenience sample of 97 Taiwanese adults who had type 2 diabetes and met the other entry criteria: $\geq 20$ years of age and able to comprehend and communicate using Mandarin or Taiwanese. The participants completed the DSMI-74 in Chinese and commented upon the items. To further establish face validity, a trained research assistant talked to the participants about their impressions of the pilot instrument.

An item analysis was used to evaluate how well each item correlated with the total score, enabling investigators to decide which items to retain (Ferketich, 1991; Nunnally \& Bernstein, 1994). First, investigators examined descriptive statistics (mean, standard deviation, and variance) for each item. An item was dropped if its mean greatly diverged from the total item mean, or if its variance was near zero. Next, investigators examined item-total correlations. Items with correlations below .3 did not sufficiently contribute to the total score; those with correlations 
above .7 probably were redundant (Ferketich). Accordingly, only items that correlated with the total score between .3 and .7 were retained. The pilot study resulted in a revised 54 item Chinese version of the DSMI that could be used in a Taiwanese population.

\section{Procedure for Data Collection}

Prior to data collection, human participants approval was obtained from The University of Michigan Institutional Review Board. In addition, permission was obtained to collect data from three hospitals in Southern Taiwan. At the request of study investigators, diabetes educators at each of the three hospitals referred potential participants from the diabetes outpatients clinic to the investigators for recruitment. Inclusion criteria were: 20 years of age or over, diagnosed with type 2 diabetes, and able to comprehend and communicate using Mandarin or Taiwanese.

\section{Sample Characteristics}

We recruited 634 Mandarin- or Taiwanese-speaking adults with type 2 diabetes from three hospitals in Southern Taiwan. Of these, $51.7 \%$ were male and $48.3 \%$ were female. Their ages ranged from 20 to 88 years $(M=59.69, S D=11.47)$. The educational levels of the sample were diverse (53.5\% with elementary school or less; $34.0 \%$ with a high school diploma; $11.5 \%$ with a college degree; and $.90 \%$ with a graduate degree). The majority of the participants $(87.2 \%)$ were married. The mean years since diagnosis were 8.46 $(S D=7.03)$. The frequency of blood glucose monitoring varied across the sample; $7.9 \%$ checked at least once daily; $50.2 \%$ checked once per month. Most of the sample $(80.8 \%)$ took oral medication for diabetes.

The 634 participants were divided into two samples based on the time of entry into the study. Sample 1 data were used for explanatory factor analysis (EFA) and confirmatory factor analysis (CFA). Sample 2 data were used for cross-validation of the confirmatory model derived from Sample 1 data.

\section{Data Analysis}

The EFA was done to assess the factor structure. Data were analyzed using with SPSS, version 12. It was completed using Equations (EQS), version 5.7, software (Bentler, 1995). EFA can identify the factor structure for a set of variables based on data instead of theory. In contrast, CFA is generally based on a strong theoretical and empirical foundation that allows the investigator to specify a hypothesized factor structure in advance and then test it (Nunnally \& Bernstein, 1994; Stevens, 1996). Thus, CFA can determine how well the proposed model fits the data (Nunnally \& Bernstein). In this study, CFA was used to examine and modify the model that emerged from the EFA. To test whether the proposed model identified by the EFA fit the data, several alternative models were tested against the hypothesized five-factor model that emerged from the EFA.

According to Noar (2003), five types of statistical models can be used to guide model testing to determine which model best fits a set of data. The following models were used in this study: (a) A null model assumes the DSMI factors are unrelated. This null model is proposed as a baseline model for comparison to other models. Several CFA fit indices rely on information provided by the null model. (b) A one-factor model tests whether the DSMI is measuring one overall factor, rather than individual factors. CFA support for this model would suggest that the phenomenon of self-management among diabetic patients is best represented by a uni-dimensional construct. (c) An uncorrelated factors model tests the idea that individual factors are independent or orthogonal. Support for this model would suggest that what is being measured in this study are independent constructs. (d) A correlated factors model tests the idea that individual factors of the DSMI are related to one another. Support for this model would suggest the possibility of a hierarchical model. (e) A hierarchical model tests the idea that a second-order factor can account for relations between individual factors. Support for this model would suggest that all factors are related to a higher-order factor. Retention of such a model would suggest that summing the total of the entire scale is appropriate and represents a meaningful and interpretable score.

To determine which of the above models best explained the data statistically, investigators used EQS 5.7 to conduct the maximum likelihood CFA using data from Sample 1. The data were treated as if they were continuous. A corrected SatorraBentler $\chi^{2}$ was used to allow for non-normality and robust standard errors for parameter estimates and robust goodness-of-fit indices. The statistical criteria used to decide which model best described the data were the Chi-square statistic $\left(\chi^{2}\right)$, the average absolute standardized residuals (AASR), the Bentler-Bonnet comparative fit indices (CFI), 
the Bentler-Bonnet non-normed fit index (NNFI), and the root mean square error of approximation (RMSEA). Additionally, the investigators used the Lagrange multiplier (LM) test to suggest alternative, better- fitting models for the data; in this test, parameters are added to see if they offer any improvement to the specified models (Bentler, 1995).

\section{RESULTS}

\section{Psychometric Testing}

Construct validity. To examine the underlying structure of the relationships among the 54 items within the DSMI, the total sample was divided into two groups. Sample $1(n=286)$ data were used to test a first-order confirmatory factor model identified in the EFA. Sample $2(n=348)$ data were used to cross-validate the results of the confirmatory factor model validated with Sample 1 data. The two samples were compared across all key demographic variables using $t$-tests. No statistically significant differences (all $p>.05$ ) were found.

\section{Exploratory Factor Analysis (EFA)}

The Sample 1 data were used to analyze the factors of the 54 diabetes self-management items. Factors were extracted using principal axis factoring and the correlation matrix and pairwise deletion method. The Kaiser-Meyer-Olkin measure of sampling adequacy was .908, indicating excellent sampling adequacy and relatively compact patterns of correlation, such that factor analysis should produce distinct and reliable factors (Field, 2000). Bartlett's test of Sphericity was significant $(p<.001)$, indicating that there were some relationships among the variables (Field, 2000). Oblique promax rotation procedures were used to rotate the factors. In examining the data from the rotated factor analysis, investigators set a criterion level of greater than .50 as the factor loading criterion needed to determine whether an item loaded on one factor or another (Costello \& Osborne, 2005). Twenty items had factor loadings less than .50; however, one of these loaded at .48, and it was retained in Factor 5 because it was conceptually consistent with that factor. Consequently, 19 items were deleted, leaving 35 items for the DSMI.
After examining the various factor solutions, investigators found that a five-factor solution provided the most meaningful factor pattern and accounted for $45.66 \%$ of the total variance. The alpha coefficients indicated good internal consistency for the composite scale $(\alpha=.93)$ and all factor-based subscales ( $\alpha$ ranged from .76 to .90). The factor structures are described as follows.

Factor 1 had 10 items, with factor loadings ranging from .55 to .73 . This factor related to patients' ability to integrate diabetes care into their daily lives through activities such as proper diet, exercise, and weight control. This factor was called self-integration.

Factor 2 had nine items, with factor loadings ranging from .51 to .90 . These items reflected patients' self-regulation of their behaviors through self-monitoring of body signs and symptoms (i.e., identifying life situations and causes related to changes in blood glucose and taking action based on these observations). Factor 2 was labeled selfregulation.

Factor 3 was termed interaction with health professionals and significant others. It contained nine items, with factor loadings ranging from .53 to .78. The common theme of these items reinforced the concept that good diabetes care involves collaboration with health care providers and significant others.

Factor 4 had four items, with factor loadings ranging from .54 to .85 . This factor specifically concerned testing blood glucose for detecting hyperglycemic or hypoglycemic reactions in order to adjust self-care activities. Factor 4 was named self-monitoring of blood glucose.

Factor 5 was composed of three items, with factor loadings ranging from .48 to .66. This factor, entitled adherence to recommended regimen, related to patients' adherence to prescribed diabetes medication and clinic visits.

\section{Confirmatory Factor Analysis (CFA)}

The validation of the DSMI-35 involved two stages of CFA using EQS 5.7. In the first stage, investigators tested whether the model identified from the EFA fit the data, and then modified it using Sample 1 data. In the second stage, this modified model was further cross-validated with Sample 2 data.

To summarize, the characteristics of the model proposed in this study are as follows: (a) the DSMI-35 has five underlying factors, (b) the five factors are intercorrelated, (c) each variable is 
assigned to only one factor, and (d) errors of measurement for each of the observed variables are independent of each other and of the factors.

Several alternative models were tested against the proposed five-factor model identified in the EFA. The overall fit indices for the four competing models improved immensely (Table 1) when comparing both the one-factor model (i.e., Model 2) and the uncorrelated factors model (i.e., Model 3 ) to the null model (i.e., Model 1). However, none of these models showed an acceptable fit.

As expected, the correlated five-factor model (i.e., Model 4) was a better fit than the uncorrelated five-factor model (i.e., Model 3); however, the overall fit indices did not reach the criteria for a good fit. To improve the five-factor model (i.e., Model 4), investigators applied the model modification tests supplied by the EQS software, including the LM test and the Wald test, to the CFA analysis of Model 4 with Sample 1 data. The Wald test indicated that no loadings should be dropped. However, the LM test suggested that additional covariance of error terms and cross-loading would improve the fit of Model 4.

Based on the largest index derived from the LM testing of Model 4, four pairs of correlated-error terms and one additional cross-loading were added to Model 4. Depending on their conceptual meaning, variables might share specific variance if they have correlated-error terms. The shared variance could be considered correlated-error variance or an identifiable additional latent variable. However, based on the conceptual meaning and the LM test, investigators decided to add correlations between errors on eight items in Model 4. In the LM test, the $\chi^{2}$ also improved when one item ["I check my blood sugar to help me make selfcare decisions (e.g., medications, diet, exercise)"], originally loading on Factor 4 (i.e., selfmonitoring of blood glucose), cross-loaded on Factor 1 (i.e., self-integration). Based on the item's conceptual meaning, it was cross-loaded on Factor 1 in Model 4.

After adding four pairs of correlated-error terms and one cross-loading item to Model 4 based on the results of the LM test and theoretical considerations, investigators tested this model again. The results showed that the modified correlated factors (i.e., Model 5), which had four pairs of correlated-error terms and one variable with cross-loading, fit the data notably better than the other models (Table 1). All fit indices indicated that Model 5 had a satisfactory goodness of fit (Satorra-Bentler scaled $\chi^{2}=950.00, \mathrm{df}=545, \chi^{2}$ / $\mathrm{df}=1.74, \mathrm{AARS}=.05, \mathrm{NNFI}=.91, \mathrm{CFI}=.91$, RMSEA $=.05)$.

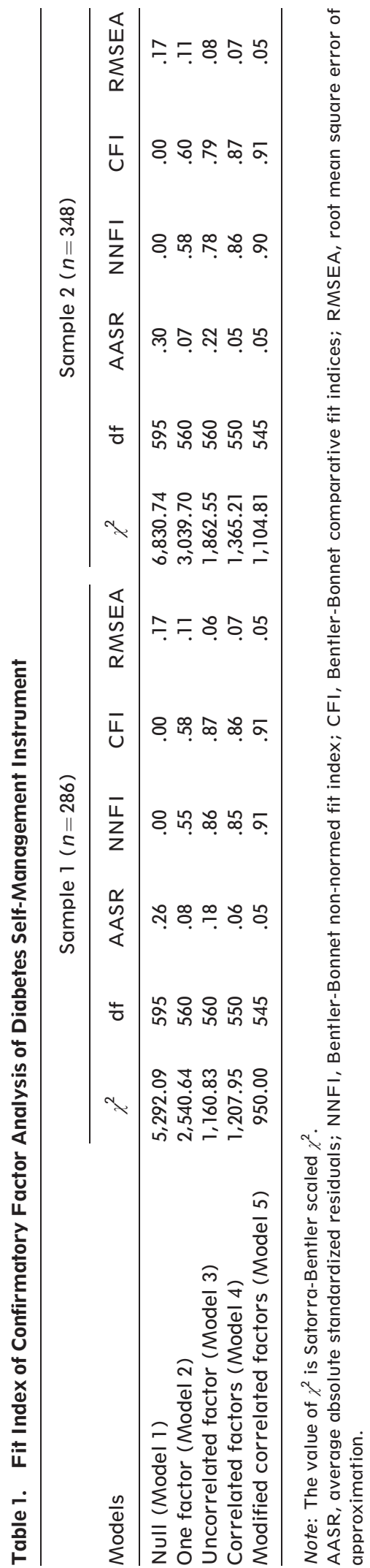




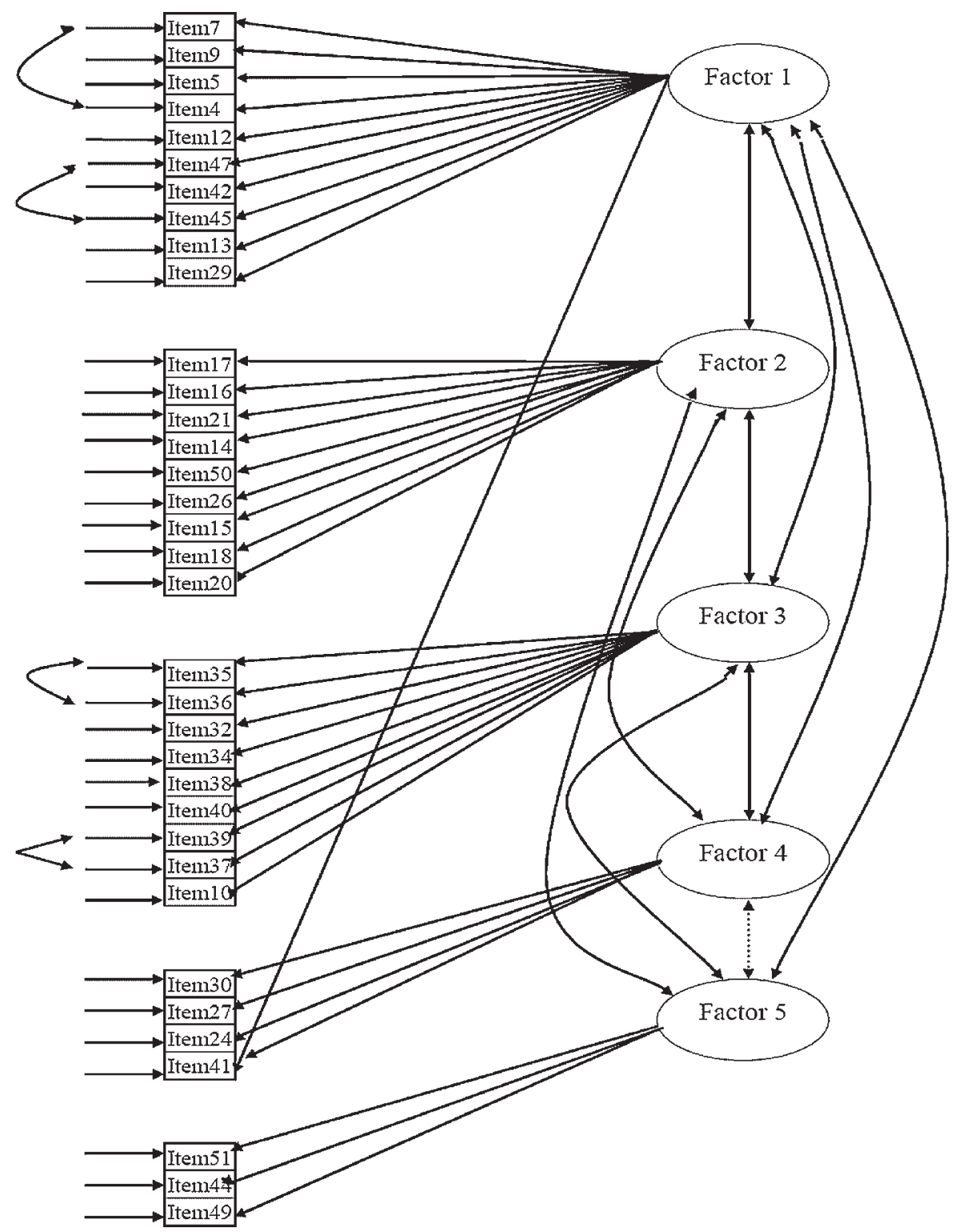

FIGURE 1. CFA model.

The final CFA model (i.e., Model 5; Fig. 1) including factor loadings and error variances is presented in Table 2. Standardized factor loading of the 35 items indicated that all factors, with loading values ranging from .45 to .98 , were statistically significant $(p<.05)$. All residual, or error, variances, ranging from .05 to .80 , were significant $(p<.05)$. The correlations between the factors, except that between Factors 4 and 5, were significant (Table 3), suggesting that an oblique solution was appropriate for this proposed model.

To evaluate the modified correlated model, Model 5, validated with Sample 1 data, investiga- tors further cross-validated this model with Sample 2 data. Fit indices demonstrated that the modified correlated model was a satisfactory fit to the data in Sample 2 (Table 1). Thus, the results of cross-validation provided further evidence for the construct validity of the DSMI with 35 items (DSMI-35).

Reliability. After factor structure confirmation, investigators used Cronbach's coefficient alpha to assess the internal consistency reliability of the total scale and factor-based subscales. Cronbach's coefficient alpha for the final version of the DSMI35 total scale was .94 . The subscale coefficient 


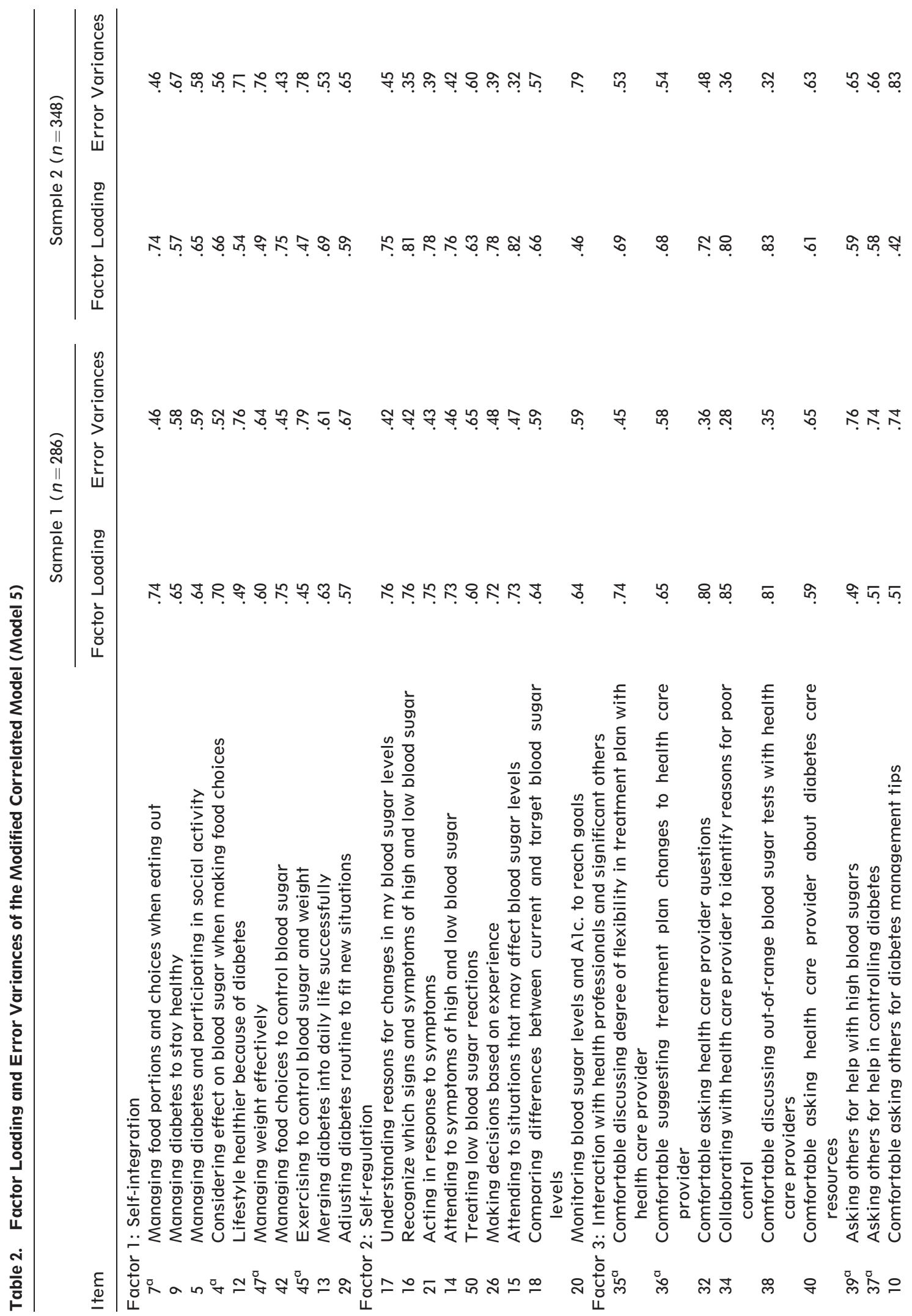




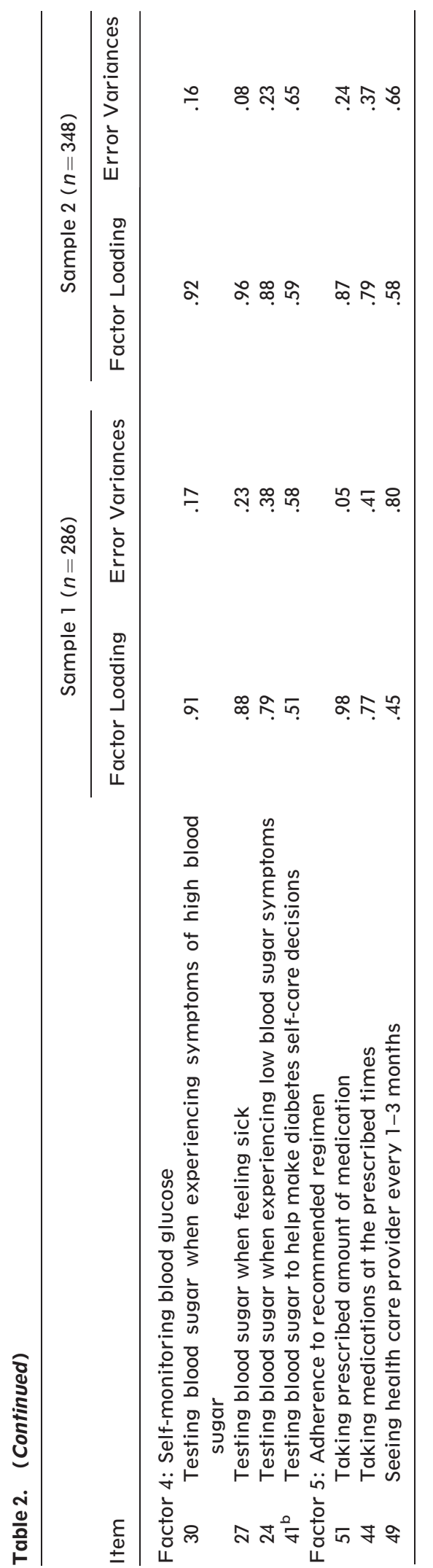

iิ

? m

荎

施 alphas ranged from .77 to .90 . The stability of the DSMI-35 over time was assessed by measuring the test-retest reliability over 2 weeks. Twenty-two participants returned the retest questionnaire DSMI-54 by mail. The final 35 item version of the DSMI examined the test-retest reliability for the total scale and subscales. The test-retest correlations for the total scale was $r=.73$, $p<.001$; each of the subscales ranged from .59 to .78

\section{DISCUSSION}

The purpose of this study was to develop, and test the psychometric properties of, a new instrument for measuring self-management behaviors among Taiwanese adults with type 2 diabetes. Investigators identified five factors from the EFA. Although these five factors represent concepts that are somewhat different from Lin's (2005) three a priori dimensions (i.e., illness adaptation, decision-making, and illness control), the five factors have a close theoretical connection to the three dimensions. For example, Factor 1 integrating diabetes care into one's life can be thought of as specific examples of illness adaptation. Factor 2 self-regulation, which is based on one's selfmonitoring, and Factor 4 using blood glucose monitoring to identify hypo and hyperglycemia are consistent with the dimension of decision making. Factor 3 concerning collaborating with health professionals and Factor 5 adherence to recommended regimen fit well with the dimension of illness control. The originally proposed three dimensions from concept synthesis may have been too broadly defined for the construct of selfmanagement. The five-factor structure and resultant five conceptual dimensions, which were verified from the empirical data, appear to be more specifically applicable to, and to reflect more of the theoretical definition of, self-management as it has been defined and used in this study. Although the EFA results indicated that the five-factor structure was sound, they provided only weak support for the construct validity of the DSMI-35. As Nunnally and Bernstein (1994) asserted, EFA should not be used to confirm factor structure because EFA is a data-driven method for exploring the factor structure of a set of variables when no theory guides the analysis.

CFA, on the other hand, is a theory-driven method. Therefore, investigators used CFA to determine whether the hypothesized model identified from EFA fit the data. They then modified the 
Table 3. Correlation Among Factors of the Modified Correlated Model (Model 5)

\begin{tabular}{|c|c|c|c|c|c|c|c|c|}
\hline \multirow[b]{2}{*}{ Factor } & \multicolumn{4}{|c|}{ Sample $1(n=286)$} & \multicolumn{4}{|c|}{ Sample $2(n=348)$} \\
\hline & $\mathrm{F} 2$ & F3 & $\mathrm{F} 4$ & F5 & $\mathrm{F} 2$ & $\mathrm{~F} 3$ & $\mathrm{~F} 4$ & F5 \\
\hline F1: Self-integration & $.71 *$ & $.54^{*}$ & $.28^{*}$ & $.26 *$ & $.75^{*}$ & $.57 *$ & $.42^{*}$ & $.47^{*}$ \\
\hline F2: Self-regulation & & $.62 *$ & $.42 *$ & $.15^{*}$ & & $.64^{*}$ & $.51^{*}$ & $.32^{\star}$ \\
\hline $\begin{array}{l}\text { F3: Interaction with health professionals and } \\
\text { significant others }\end{array}$ & & & $.43^{*}$ & $.15^{*}$ & & & $.41^{*}$ & $.29 *$ \\
\hline F4: Self-monitoring of blood glucose & & & & -.03 & & & & .04 \\
\hline
\end{tabular}

${ }^{*} p<.05$.

model accordingly. The CFA results continued to support the five factors of the DSMI-35. While the initial fit indices did not provide full support for this model, a modified five-factor correlated model resulted in better-fit indices. Moreover, this modified model cross-validated data from the second sample, providing stronger support for construct validity of this newly constructed DSMI-35. It appears that the subscales based on the five firstorder factors could be scored individually. Yet, the correlated factors suggested the existence of a higher-order latent variable that subsumes all five factors, presumably self-management.

The homogeneity or uni-dimensionality of items is a major issue in assessing the psychometric properties of an instrument. The Cronbach's alpha coefficients for the DSMI-35 total scale (.94) and each of the five subscales (.77-.90) indicated good internal consistency for this newly constructed instrument. However, the high Cronbach's alpha coefficient for the DSMI-35 total scale implied that there may be some redundancy of items. Devellis (1991) suggested that alpha coefficients greater than .90 may indicate the need to shorten the instrument length. Future studies are needed to examine whether some items can be combined or deleted.

Overall, the results of test-retest analysis suggested that the DSMI-35 and four of its subscales were relatively stable over a 2 -week period. The subscale of self-monitoring of blood glucose had a moderately low test-retest correlation $(r=.59)$. This could be attributed to the characteristics of the study participants-half of the participants reported that they checked their blood glucose only once per month, during their monthly hospital visit. This was because they had no equipment at home. That is, often patients' self-monitoring of their blood glucose depends on whether the equipment for checking blood glucose is available. The low test-retest correlation may also have been a result of participants' attitudes toward self monitoring of blood glucose. It is common for diabetic patients to judge levels of blood glucose by their own body sensations or signals such as cold sweating, thirst. In addition, they may judge their blood glucose levels by their previous experiences of the effects of diet, exercise, and stress on blood glucose. In short, since self monitoring of blood glucose may vary with patients' attitudes, schedules, or circumstances, the low retest reliability of the subscale of self monitoring of blood glucose may have been due to the instability of the behavior rather than the scale. Further test-retest studies are warranted.

This study provides evidence to support the content, face, and construct validity as well as the internal consistency and retest reliability of the DSMI-35 in a Taiwanese population. The DSMI35 should be tested in other patient populations since there are differences in language, culture, and health systems, including patient and health care provider expectations and methods of selfmanagement implementation. Because the initial draft of this instrument was in English, tests of the psychometric properties of the DSMI-35 in a population of English-speaking patients should be easier to conduct.

Self-management is a complex concept. Before clinical questions about self-management can be investigated and answered, there must be valid and reliable measures that provide empirical data. As more attention is given to patients' management of their illnesses, health care professionals can provide improved care by better understanding patients' self-management behaviors. The DSMI developed in this study can be used to assess how patients with type 2 diabetes take care of themselves; to develop more relevant, patient-centered teaching materials; and to implement interventions tailored to the needs of individual patients.

\section{REFERENCE}

Anderson, R.M., \& Robins, L.S. (1998). How do we know? Reflections on qualitative research in diabetes. Diabetes Care, 21, 1387-1388. 
Barlow, J., Wright, C., Sheas, J., Turner, A., \& Hainsworth, J., (2002). Self-management approaches for people with chronic conditions: A review. Patient Education and Counseling, 48, 177-187.

Bentler, P.M. (1995). EQS structural equations program manual. Encino, CA: Multivariate Software, Inc.

Carlson, E.D. (2000). A case study in translation methodology using the health-promotion lifestyle profile II. Public Health Nursing, 17, 61-70.

Centers for Disease Control and Prevention. (2005). Number of Americans with diabetes continues to increase. Retrieved July 7, 2006, from http://www. cdc.gov/od/oc/media/pressrel/fs051026.htm.

Costello, A.B., \& Osborne, J.W. (2005). Best practices in exploratory factor analysis: Four recommendations for getting the most from your analysis. Practical Assessment Research and Evaluation, 10, 1-9. Retrieved July 31, 2007, from http://pareonline. net/pdf/v10n7.pdf.

Devellis, R.R. (1991). Scale development: Theory and application. Newbury Park, CA: Sage.

Fain, J.A., Nettles, A., Funnell, M.M., \& Prochownik, D.C. (1999). Diabetes patient education research: An integrative literature review. The Diabetes Educator, $25,7-15$.

Ferketich, S. (1991). Focus on psychometrics: Aspects of item analysis. Research in Nursing and Health, 14, $165-168$.

Field, A. (2000). Discovering statistics using SPSS for Windows. London: Sage.

Funnell, M.M., \& Anderson, R.M. (2000). The problem with compliance in diabetes. JAMA, 284, 1709.

Gonder-Frederick, L.A., Cox, D.J., \& Ritterband, L.M. (2002). Diabetes and behavioral medicine: The second decade. Journal of Consulting and Clinical Psychology, 70, 611-625.

Hill-Briggs, F. (2003). Problem solving in diabetes selfmanagement: A model of chronic illness selfmanagement behavior. Annals of Behavioral Medicine, 25, 182-193.
Lin, C.C. (2005). Development and testing of the diabetes self-management instrument. Unpublished doctoral dissertation, The University of Michigan.

Lin, C.C., Anderson, R.M., Hagerty, B.M., \& Lee, B.O. (in press) Diabetes self-management experience: A focus group study of Taiwanese patients with type 2 diabetes. Journal of Clinical Nursing.

Lynn, M.R. (1986). Determination and quantification of content validity. Nursing Research, 35, 382-385.

Noar, S.M. (2003). The role of structural equation modeling in scale development. Structural Equation Modeling, 10, 622-647.

Nunnally, J.C., \& Bernstein, I.H. (1994). Psychometric theory. New York: McGraw-Hill, Inc.

Petrie, K.J., Broadbent, E., \& Meechan, G. (2003). Selfregulation interventions for improving the management of chronic illness. In L.D. Cameron \& L. Howard (Eds.), The self-regulation of health and illness behaviour (pp. 257-277). London: Routledge.

Price, M.J. (1993). An experiential model of learning diabetes self-management. Qualitative Health Research, 3, 29-54.

Schilling, L.S., Grey, M., \& Knafl, K.A. (2002). The concept of self-management of type I diabetes in children and adolescents: An evolutionary concept analysis. Journal of Advanced Nursing, 37, 87-99.

Stevens, J. (1996). Applied multivariate statistics for the social sciences (3rd ed.) Hillsdale, NJ: Erlbaum.

Taiwan National Department of Health. (2004). Annual medical statistics in Taiwan in 2004. Retrieved July 17, 2006, from http://www.doh.gov.tw/statistic.

Waltz, C.F., Strickland, O.L., \& Lenz, E.R. (2005). Measurement in nursing and health research (3rd ed.) New York: Springer.

Whittemore, R. (2000). Strategies to facilitate lifestyle change associated with diabetes mellitus. Journal of Nursing Scholarship, 32, 225-232.

World Health Organization. (2004). Global strategy on diet, physical activity and health. Retrieved January 25, 2007, from http://www.who.int/dietphysicalactivity/ strategy/eb11344/strategy_english_web.pdf. 